\title{
A Bidimensional Photon-counting Microchannel Plate Detector Using a Wedge and Strip Anode
}

\author{
R. Drazdys, J. Jukonis, A. Skrebutènas, V. Vansevičins and \\ G. Vilkaitis \\ Institute of Physics, Goštauto 12, Vilnius 2600, Lithuania
}

\begin{abstract}
A photon-counting detector with a $45 \mathrm{~mm}$ diameter active area has been developed using a microchannel plate (MCP) and the wedge and strip readout system. The detector uses a UV transparent glass input window proximity coupled to a stack of MCP. Quantum efficiency (QE) of the detector photocathode is $\sim 20 \%$. The resolution of $\sim 60 \mu \mathrm{m}$ FWHM at a gain of $5 \cdot 10^{7}$ has been achieved.
\end{abstract}

\section{Introduction}

The paper describes the performance characteristics of a microchannel plate photon-counting detector developed at the Astrophysical Instrumentation Laboratory of the Institute of Physics, Vilnius, Lithuania.

\section{Detector}

A schematic view of the detector is shown in Fig. 1. Its first component is a photocathode where an incident photon may be transformed into electron. The electron crosses the $0.5 \mathrm{~mm}$ gap and is received by a stack of microchannel plates with the $15 \mu \mathrm{m}$ channel diameter and the length to diameter ratio of 55:1. The MCP stack consists of two "V" configuration stacks separated by a $1 \mathrm{~mm}$ gap. MCPs in both "V" stacks are separated by a $15 \mu \mathrm{m}$ electric contact rins.

As a result of traversing through the MCP stack, photoelectrons are multiplied by a factor of up to $\sim 10^{8}$, depending on the voltage applied across the stack. The electron cloud subsequently drifts through a region of uniform electric field to the wedge and strip anode. On this way the electron cloud grows to the size more than one repetition period of the wedge and strip anode pattern.

The wedge and strip anode (Martin et al., 1981) uses three electrods to divide the charge proportionally to $X$ and $Y$ coordinates of the event. The width of the wedges and strips varies linearly with $Y$ and $X$, and the coordinates of the event may be deduced from the following simple algorithm

$$
X=\frac{2 Q_{S}}{Q_{W}+Q_{S}+Q_{Z}}, \quad Y=\frac{2 Q_{W}}{Q_{W}+Q_{S}+Q_{Z}}
$$




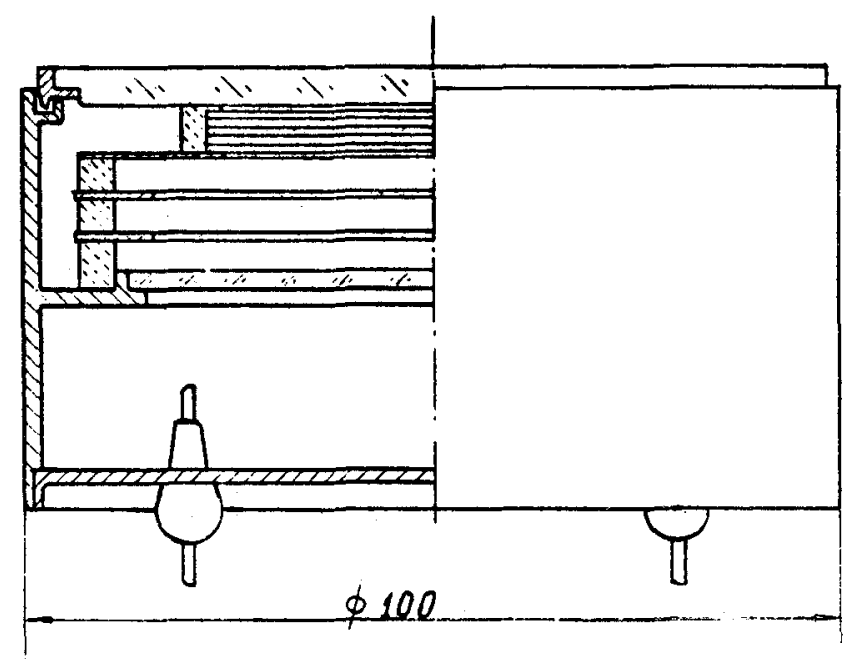

Figure 1. A cross view of the detector.

here $Q_{W}, Q_{S}$ and $Q_{Z}$ are the amounts of charge falling on the $\mathrm{W}, \mathrm{S}$ and $\mathrm{Z}$ electrodes, respectively.

\section{Electronics}

The electric signals received at $\mathrm{W}, \mathrm{Z}$ and $\mathrm{S}$ electrodes are amplified and shaped and then are passed to a signal processing unit which produces 10 bit addresses corresponding to $X$ and $Y$ coordinates of the event.

The data acquisition system is based on a PC/AT computer and uses a $1024 \times 1024$ video memory, allowing real time display of information. The video memory can store $2 \mathrm{MB}$, and each memory element can register up to 65000 events. Memory contents is displayed in real time on a monitor.

\section{Test results}

Quantum efficiency. The quantum efficiency (QE) of a bialkali cathode, combined with the UV transparent glass transmission, at the peak is approximately $20 \%$. The detective quantum efficiency (DQE) of the detector is limited by the photoelectron detection efficiency of the unfilmed MCP, typically yielding $\sim 60 \%$ (Clampin et al., 1988).

Position resolution. Principal factors affecting the spatial resolution of the detector are the electronic noise attributed to the amplifiers, the partition noise caused by the statistical deviation in the charge division among the three electrodes (Siegmund et al., 1983) and the lateral spread of the photoelectron cloud incident on the MCP (Clampin et al., 1988).

The experimental resolution was determined by imaging a resolution test chart. 
The analysis of the bar pattern of one dimensional profiles yields the resolution of the order of $60 \mu \mathrm{m}$ FWHM.

Image distortion and counting rate. The image linearity of the detector has been assessed by examination of a resolution test chart image. The most noticeable barrel-type distortion is observed in a few mm zone at the edge of the image. This distortion is attributable to variations in the field strength between the MCP and the anode.

The counting rate of the overall system, $5 \cdot 10^{4}$ counts per second, was limited by electronics.

\section{Conclusions}

The described detector is ready for tests in astronomical observations. We hope that this will be a highly competitive instrument for different low light level astronomical and other applications.

\section{References:}

Martin C., Jelinsky P., Lampton M., Malina P.F. and Anger H.O. 1981, Rev. Sci. Instrum., 52(7), 1067.

Clampin M., Crocker J., Paresce F., and Rafal M. 1988, Preprint of STScI, No. 276, 5.

Siegmund O.H.W, Clothier S., Thornton J., Lemen J., Harper R., Mason I.M., and Culhane J.L. 1983, IEEF Trans. Nucl. Sci., Vol. NS-30, No.1, 503.

Chakrabarti S., Siegmund O.H.W., and Hailey C. 1988, in Instrumentation for GroundBased Optical Astronomy, ed. Robinson L.B., Springer - Verlag, 574. 